Revista Economía Y Administración, Vol. 12, N o 1, 2021

\title{
ANÁlisis DEL impacto DEL DESEMPLEO POR SEXO EN EL CRECIMIENTO ECONÓMICO DE HONDURAS PARA EL PERÍODO
} 1991-2019

\author{
Israel Alberto Rivera Posadas* \\ Keltsin Lossue Lagos Trejo** \\ Allan Mauricio Ardón Montalván*** \\ Nahomy Paola Cruz Galeas ${ }^{* * * *}$
}

DOI: I0.5377/EYA.VI2II.I2964

Recibido: 19/05/2021 Aceptado: 30/11/2021

\section{RESUMEN}

Este documento examina la relación entre la producción y el desempleo a la luz de la Ley de Okun por sexo para Honduras durante el periodo 1991 - 2019. Se emplea por medio de una regresión simple del modelo de primeras diferencias y estimado por $\mathrm{MCO}$, esto a través de tres enfoques: desde la población total, la población masculina y la femenina.

Los resultados de los tres enfoques establecen el signo negativo del coeficiente de Okun como evidencia de la relación inversa entre variaciones del PIB y la tasa de desempleo, en el cual la población masculina es la más afectada por variaciones del PIB debido a una mayor magnitud del coeficiente de Okun, explicado por la estructura laboral hondureña, donde las actividades económicas más relevantes, poseen una mayor participación masculina. Sin embargo, estos coeficientes no son estadísticamente significativos, incumpliendo la ley de Okun para la economía hondureña. Esto implica la no aplicación del modelo para la economía del país como la consideración de más variables que expliquen la causalidad de la tasa de desempleo en un mercado laboral marcado por la informalidad y el subempleo.

PALABRAS CLAVES: ley de Okun, desempleo, y PIB.

JEL: J01, J71

\footnotetext{
*Investigador,Universidad Nacional Autónoma de Honduras,https://orcid.org/0000-0002-5424-8779,Correo electrónico:israelalbertor2000@ gmail.com, Tegucigalpa, Honduras

** Investigador, Universidad Nacional Autónoma de Honduras, https://orcid.org/0000-0003-4319-1663, Correo electrónico: keltsinlagos@ gmail.com,Tegucigalpa, Honduras

****Investigador, Universidad Nacional Autónoma de Honduras, https://orcid.org/0000-0003-0245-8704, Correo electrónico: alpok1977@ gmail.com Yuscarán, Honduras.

******* Investigadora, Universidad Nacional Autónoma de Honduras, https://orcid.org/0000-0002-8736-9235, Correo electrónico: naho. galeas20@gmail.com Tegucigalpa Honduras.
} 


\section{THE IMPACT OF UNEMPLOYMENT ON ECONOMIC GROWTH IN} HONDURAS: FROM A SEX PERSPECTIVE

1991-2019

Israel Alberto Rivera Posadas*

Keltsin Lossue Lagos Trejo**

Allan Mauricio Ardón Montalván***

Nahomy Paola Cruz Galeas ${ }^{* * * *}$

\section{DOI: IO.5377/EYA.VI2II.I2964}

Recived: 19/05/2021 Accepted: 30/11/2021

\section{ABSTRACT}

This paper examines the relationship between production and unemployment in light of Okun's Law by sex for Honduras, during the period 1991 - 2019. It is employed by means of a simple regression of the first differences model and estimated by OLS, this through three approaches: from the total population, the male and female population.

The results of the three approaches establish the negative sign of the Okun coefficient as evidence of the inverse relationship between variations in GDP and the unemployment rate, in which the male population is the most affected by variations in GDP due to a greater magnitude of the Okun coefficient, explained by the Honduran labor structure, where the most relevant economic activities have a greater male participation. However, these coefficients are not statistically significant, not complying with Okun's law for the Honduran economy. This implies the non-application of the model for the country's economy, as well as the consideration of more variables that explain the causality of the unemployment rate, in a labor market marked by informality and underemployment.

KEYWORDS: Okun's law, unemployment, and GDP.

JEL: J01, J71

\footnotetext{
* Researcher, National Autonomous University of Honduras https://orcid.org/0000-0002-5424-8779 Email: israelalbertor2000@gmail.com, Tegucigalpa Honduras

** Researcher, National Autonomous University of Honduras, https:/orcid.org/0000-0003-4319-1663, Email: keltsinlagos@gmail.com, Tegucigalpa Honduras

*** Researcher, National Autonomous University of Honduras, https://orcid.org/0000-0003-0245-8704, Email: alpok1977@gmail.com Tegucigalpa, Honduras

***** Researcher, National Autonomous University of Honduras, https://orcid.org/0000-0002-8736-9235, Email: naho.galeas20@gmail.com Yuscarán, Honduras
} 


\section{INTRODUCCIÓN}

La dinámica del mercado laboral, en particular el análisis del desempleo de las mujeres es relevante dadas las fluctuaciones de este mercado laboral. Para el caso de Honduras, es de destacar que es el segundo país de Centroamérica que presenta la mayor tasa de desempleo femenino con 8.1\%, por debajo de Costa Rica que presenta una tasa de $15 \%$ para el año 2019. Aunado a lo anterior, la tasa de desempleo de las mujeres se ha situado por encima de la tasa de desempleo de los hombres, lo cual refleja la asimetría de la brecha de desempleo según el sexo existente (Instituto Nacional de Estadística, sf).

Es preciso mencionar que en Honduras las investigaciones que abordan el análisis del mercado laboral desde el punto de vista del sexo son escasos. En la literatura se menciona la investigación de Méndez et al, (2020) que busca evidenciar las brechas salariales que enfrentan las mujeres. Sin embargo, no existen investigaciones que analicen la relación entre el producto y las tasas de desempleo (conocido teóricamente como la ley de Okun) específicamente para Honduras.

La ley de Okun muestra la relación inversa entre la variación del producto y la variación de la tasa de desempleo. Lo que busca demostrar esta ley es que en periodos de crecimiento económico se debería incrementar el nivel de empleo en todos los sectores y a su vez disminuir la tasa de desempleo económico, ello debido a que el nivel de producto depende en gran medida de la mano de obra utilizada en el proceso productivo (Paez, 2013).

El presente documento busca examinar la relación del producto y la tasa de desempleo por sexo para la economía de Honduras utilizando información estadística del Banco Mundial para el periodo comprendido entre los años 1991-2019. Se emplea el análisis de regresión simple utilizado para analizar la ley de Okun por primeras diferencias. Una vez estimados los modelos se procederá a comparar los resultados obtenidos con el modelo general para la economía hondureña.

El resto de la investigación se organiza de la siguiente manera: en la sección dos, se realiza una revisión a la literatura; el tercer aparado, plantea la metodología que se abordará; en la cuarta sección del documento, se muestran los principales resultados y; finalmente en la quinta sección, se exponen las conclusiones del estudio.

\section{MARCO TEÓRICO}

La relación entre la tasa de desempleo y el producto interno bruto es señalada por (Franco, 2017), a través de la descripción de las formas de desarrollo empírico de la ley de Okun, cuyo análisis presenta las tres aproximaciones realizadas por Okun. La primera comprende la aplicación del modelo de primeras diferencias, que permite analizar la influencia de las variaciones en la tasa de desempleo $(\Delta \mathrm{Ut})$, y su relación con el comportamiento anual del PIB real $(\Delta \mathrm{Yt})$. La segunda está determinada por la aproximación del modelo de brechas, el cual hace una relación de la tasa de desempleo, con la brecha entre la tasa de crecimiento potencial y la real. En la tercera y última demostración empírica, se utilizan los ajustes de tendencia y elasticidad, para realizar la medición de la relación entre la tasa de ocupación efectiva y la de pleno empleo. 
El desarrollo empírico de las ecuaciones de la ley de Okun fue expresado por (Franco, 2017), de la siguiente manera:

1. Modelo de primeras diferencias

$$
\begin{aligned}
& U_{t}-U_{t-1}=\beta_{0}+\beta_{1}\left(Y_{t}-Y_{t-1}\right)+\varepsilon_{t} \\
& \Delta U_{t}=\beta_{0}+\beta_{1}\left(\Delta Y_{t}\right)+\varepsilon_{t}
\end{aligned}
$$

2. Modelo de brechas

$$
\begin{aligned}
& U_{t}-U_{t}^{*}=\beta_{0}+\beta_{1}\left(Y_{t}-Y_{t}^{*}\right)+\varepsilon_{t} \\
& \Delta U_{t}^{*}=\beta_{0}+\beta_{1}\left(\Delta Y_{t}^{*}\right)+\varepsilon_{t}
\end{aligned}
$$

\section{Ajuste de tendencia y elasticidad}

$$
\ln \left(E_{t}\right)=\beta_{0}+\beta_{1} \ln \left(Y_{t}\right)+\beta_{2} t+\varepsilon_{t}
$$

De acuerdo con Paez (2013), para el caso de Latinoamérica expone el análisis de Argentina, Brasil, Chile, México, Uruguay y Venezuela donde señala que las fuertes contracciones del PIB van acompañadas de disminuciones en la tasa de empleo que se revierten con las respectivas recuperaciones, en donde de manera amplia realiza el estudio de la ley de Okun evaluando otros factores además de la tasa de empleo, como la influencia de los precios de los factores (capital y trabajo).

Desde una perspectiva más específica, una de las demostraciones en base a la Ley de Okun es aplicada para el caso de Centroamérica en el estudio de Correa y Cervantes (2017), donde según estadísticas la variación del producto y la tasa de desempleo en el periodo de estudio comprendido entre los años 1992-2016 para Costa Rica, señala que las variaciones en la tasa de crecimiento del PIB son explicadas en un $45 \%$ por los cambios en la tasa de desempleo. Añaden que sí existe la relación inversa que confirma que un aumento del 1\% en el PIB real se expresa como una caída en la tasa de desempleo igual al $0.22 \%$.

A nivel internacional estos planteamientos sobre el nivel de empleo son observados por Gálvez y Rodríguez (2013) a través de una perspectiva de hombres y mujeres al analizar la condición del empleo durante las recesiones en los últimos cien años para el caso de España; donde concluyen que, pese a la intensificación del trabajo de las mujeres, las tasas de empleo de los hombres se recuperan antes de los periodos de crisis económica. Además, se identificó que el empleo de las mujeres posterior a la crisis tiene características más inestables en comparación al periodo anterior a la crisis.

Para complementar la información respecto al desempleo bajo este enfoque, Chamberlin (2011), desarrolla una de las principales investigaciones del mercado laboral, en donde define que los movimientos entre estar empleado y desempleado durante las recesiones económicas son más perjudiciales para el sexo masculino que para el femenino, esto debido a que el empleo femenino tiende a concentrarse más en sectores laborales más estables, como los servicios públicos.

En términos de política económica la regularización de Okun es importante, ya que aporta información sobre los efectos que generan las variaciones cíclicas de la actividad económica sobre el desempleo, o visto desde otro ángulo, en cuánto se aleja el producto de su nivel potencial cuando existen en la economía recursos ociosos. 
Loria (2012), apoyado en una regresión de tipo UVAR, permitió observar cómo, en el caso de la economía mexicana, la estabilidad de los crecimientos económicos se ven el doble de reflejado en el sexo masculino. La evidencia de Loria solo es posible por las condiciones de la economía mexicana compuesta productivamente por gran cantidad de mano de obra masculina.

De acuerdo a Weller (2012), cabe analizar los factores que son aplicables para demostrar la ley de Okun para el caso de Latinoamérica, considerando todas las variables que inciden en el mercado laboral. Y recordando el planteamiento de Knotek, los resultados dependen de la estabilidad y los ciclos económicos de estas economías.

Sin embargo, desde el desarrollo inicial de Okun y dadas las nuevas técnicas de validación econométricas, diversos autores han realizado una serie de críticas, entre la más destacables se identificaron:

\section{Regla empírica}

La aplicación de la ley de Okun ha sido verídica para ciertas economías, observando los movimientos del GAP desde diferentes estudios. No obstante, Knotek concluye que: primero, no es una relación rigurosa debido a las varias excepciones que se han registrado de la ley de Okun, esto para hacer recordatorio que es una regla empírica y no una ley como su connotación dice; segundo, que no es estable en el tiempo y depende del ciclo económico, si es momento de recesión o expansión para lograr obtener mayor veracidad (2007).

\section{Bidireccionalidad del modelo}

El planteamiento original de Okun tomaba el valor inverso del coeficiente del producto para encontrar el efecto de la tasa de desempleo en el crecimiento económico. Sin embargo, Barreto y Howland (citado en Pérez et al., 2014), critican el interpretar la relación producto-desempleo en ambos sentidos, ya que afirman que el coeficiente de Okun tiene una sola lectura. El analizar dicho efecto causal requeriría la especificación de otro modelo econométrico donde la variable endógena sea el producto.

\section{Variables Explicativas}

Okun al considerar solamente en su estudio a la producción como variable explicativa del nivel desempleo, excluye variables que podrían otorgar un mayor nivel de explicación al modelo. Prachowny (citado en Sánchez-Fung, 2015) afirma que deberían ser consideradas variables como: la fuerza de trabajo, la productividad y las horas de trabajo.

Considerando las críticas mencionadas, las investigaciones aplicando la ley de Okun como referencia para la comparación del comportamiento de los ciclos y variaciones de crecimiento económico y la tasa de desempleo nacional, son ampliamente utilizados para análisis de los países de los diferentes continentes, entre ellas se muestran las aplicaciones de la relación encontrada para Europa y algunos países de América Latina.

En el caso de Europa los diferentes modelos de la ley de Okun demuestran claramente la relación inversa entre el output gap y el cambio en la tasa de paro laboral tal como lo da a conocer Castellano (2013), con su aplicación de la ley de Okun a España, Suecia e Inglaterra tomando datos del Eurostat (Oficina Europea de Estadística). El estudio determinó que la ley de Okun es verídica en estas economías y que además se ve afectada por la edad y el sexo de los individuos y no solo por los crecimientos económicos. Se demostró además como los jóvenes son más adversos a los riesgos en los periodos de crisis económicas especialmente los hombres que poseen una correlación negativa muy alta en las regresiones respecto al desempleo. 
Castellano, utilizó el método de relación de brechas $U=\bigotimes+ß(G A P)$ donde son de gran importancia el PIB potencial y el PIB ajustado, para estimar la brecha de producto GAP que ayudan a encontrar la variación de la tasa de desempleo U.

Una clara forma de interpretar estas diferencias encontradas por Castellanos es mediante la aplicación de $\beta$ como la medida de crecimiento de la productividad, colocando a la variación del empleo entre la variación de la producción que es la inversa del PIB por trabajador, por tanto, si se descubre muy alto significa que la economía crecerá gracias al incremento del empleo y un menor crecimiento de la productividad como se muestra en el caso de España. Para Suecia e Inglaterra esta correlación negativa es menor y una caída del PIB solo logra un pequeño aumento de la tasa de desempleo.

Otro estudio importante, en el caso del viejo continente, en gran medida por sus conclusiones Pérez et al, (2014), evaluó la relación de causalidad entre las variables para el caso de España para luego cuantificarlas y posteriormente analizar la existencia de diferencias significativas entre hombres y mujeres. Con una base trimestral de 1996-2013, estima un modelo dinámico de brechas diferido por sexo; determinando que la ley de Okun es verídica para la economía española.

Los resultados generales para el país indican que por cada variación de $1 \%$ en el producto se produce una desviación respecto a su tendencia de -0.307 puntos porcentuales en el corto plazo y -0.865 en el largo plazo. $\mathrm{Si}$ se diferencia el resultado por sexo se obtiene que el efecto en los hombres es de -0.216 y -0.813 y para las mujeres de -0.423 y -0.905 . Cabe resaltar que el sexo femenino es quien más se ve afectado en el corto plazo y el sexo masculino en el largo plazo.

Finalmente, los autores Perez et al, realizan un estudio de asimetría entre las brechas de desempleo de hombres y mujeres respecto al ciclo económico, concluyendo que en periodos de expansión la tasa de desempleo de las mujeres suele situarse por encima de la de los varones, y en periodos de recesión se aprecia una disminución de la brecha entre hombres y mujeres, es decir, surge un ajuste de las tendencias de las tasas de desempleo.

Dentro de los estudios de América Latina específicamente para México en Loria, Libreros y Salas (2012) estiman dos modelos mutuamente complementarios, el primero estimado mediante Mínimos Cuadrados Ordinarios MCO y el segundo mediante un modelo de vectores autorregresivos irrestrictos (UVAR por sus siglas en inglés) con impulsos generalizados encontró una relación causal negativa entre las variables, cumpliendo así la ley de Okun. De igual manera, encontró que el coeficiente de desempleo masculino (42.48) tiene el doble de magnitud que el desempleo femenino (22.27), evidenciando la segregación en el empleo.

Mediante el análisis impulso respuesta, Loria Et al., observan un rezago en la tasa de desempleo femenino, a diferencia de la tasa de desempleo masculina el cual el efecto dinámico es inmediato, esto se debe principalmente a la mayor participación masculina en el sector formal (2012).

Asimismo, es importante recalcar las características relevantes del mercado laboral mexicano donde al igual que el resto de las economías latinoamericanas Correa y Cervantes (citado en Peñaherrera, 2012) poseen una recalcada fragilidad y baja productividad, sufren un desempleo estructural, provocando en el caso de México una mayor informalidad en las mujeres y una mayor propensión al subempleo en los hombres.

Entre los estudios realizados en Latinoamérica para comprobar la Ley de Okun, se encuentra la evaluación para el caso de Colombia desde una perspectiva de hombres y mujeres, para el periodo 2008-2017. En donde se 
hace una estimación de la Ley de Okun desde la metodología de brechas llevando a cabo los respectivos análisis del filtro de Hodrick y Prescott y de cointegración multivariada.

Los hallazgos en su evidencia empírica muestran la valoración de los ciclos económicos además de las dinámicas internas del mercado laboral, y en esa misma lógica los procesos "indican que las mujeres son más sensibles a los ciclos económicos que los hombres en el mercado laboral” (Sanchez \& Gutierrez, 2018). Aunado a la estimación del caso de España, también señalan el alza del desempleo en los hombres durante periodos de recesión, y el incremento para las mujeres en momentos de expansión económica.

Entre los resultados obtenidos de la segregación sectorial por sexo, a través de la aplicación de una metodología descriptiva, correlacional y no experimental y la aplicación del modelo de brechas; Gutierrez \& Sanches (2018), determinan una relación positiva entre producto- brecha de desempleo por género; donde al haber una disminución de un billón de pesos en el producto interno bruto (PIB) sobre la brecha de desempleo se generará una disminución de 0.1622 puntos porcentuales en la brecha de desempleo por género en promedio.

\subsection{Hechos ESTILIZADOS PARA HONDURAS}

Los trabajos referentes a la aplicación de la ley de Okun para el caso de Honduras son escasos. Sin embargo, un estudio realizado recientemente por Martin \& Porras, señala la relación desempleo-producto para el caso Latinoamericano; en base a las características particulares que presenta el mercado laboral en las economías en vías de desarrollo. La aplicación econométrica se realiza a través de Mínimo Cuadrados Ordinarios considerando, que tanto las variables como los componentes cíclicos son variables estacionarias (Martin \& Porras, 2020, p. 13).

En la evaluación para Honduras, los resultados obtenidos indican la no significancia de los coeficientes, dado que el mismo estudio señala que los valores son cercanos a cero oscilando en un promedio entre 0,1 y 0,12 (2020, p. 16). En este sentido, se llevará a cabo una nueva valoración de la tasa de desempleo por sexo para analizar su comportamiento a lo largo del tiempo considerando datos que muestran la dinámica del mercado laboral hondureño.

Figura 1. Tasa de desempleo total y por sexo para Honduras 1991-2019

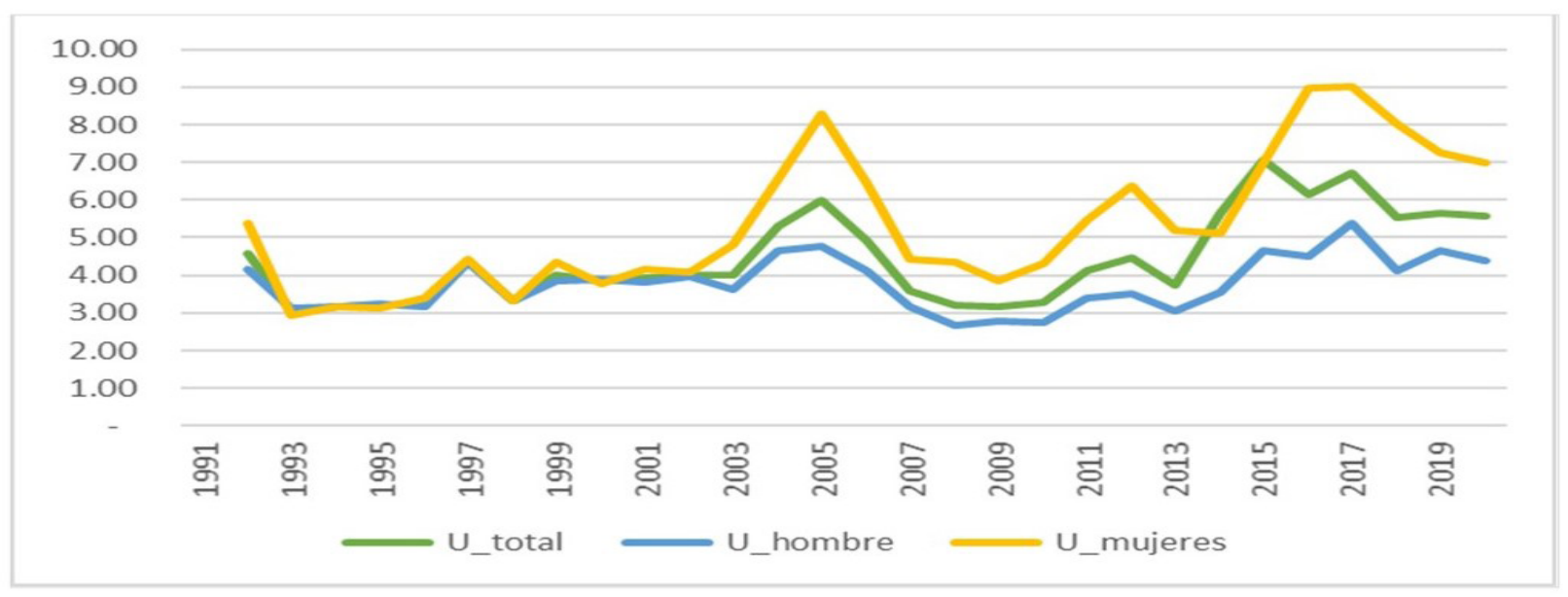

Fuente: Elaboración propia con datos del Banco Mundial (BM). 
La Figura 1, muestra las constantes fluctuaciones y la brecha de desempleo existente en el país; el comportamiento de la brecha ocupacional, explica como en los periodos de crisis las mujeres presentan mayores tasas de desempleo, pero son los hombres los que en forma relativa reciben los golpes más duros por ser la población más empleada como evidentemente se puede apreciar entre los años 1998-2002, período caracterizado por la recuperación de los sectores económicos luego del declive comercial por el paso del Huracán Mitch. Estos impactos se repiten nuevamente en el año 2009, explicado por la inestabilidad sociopolítica, donde los niveles de empleo femenino y masculino disminuyen significativamente.

Figura 2. Variación de la tasa de desempleo por sexo para Honduras 1991-2019

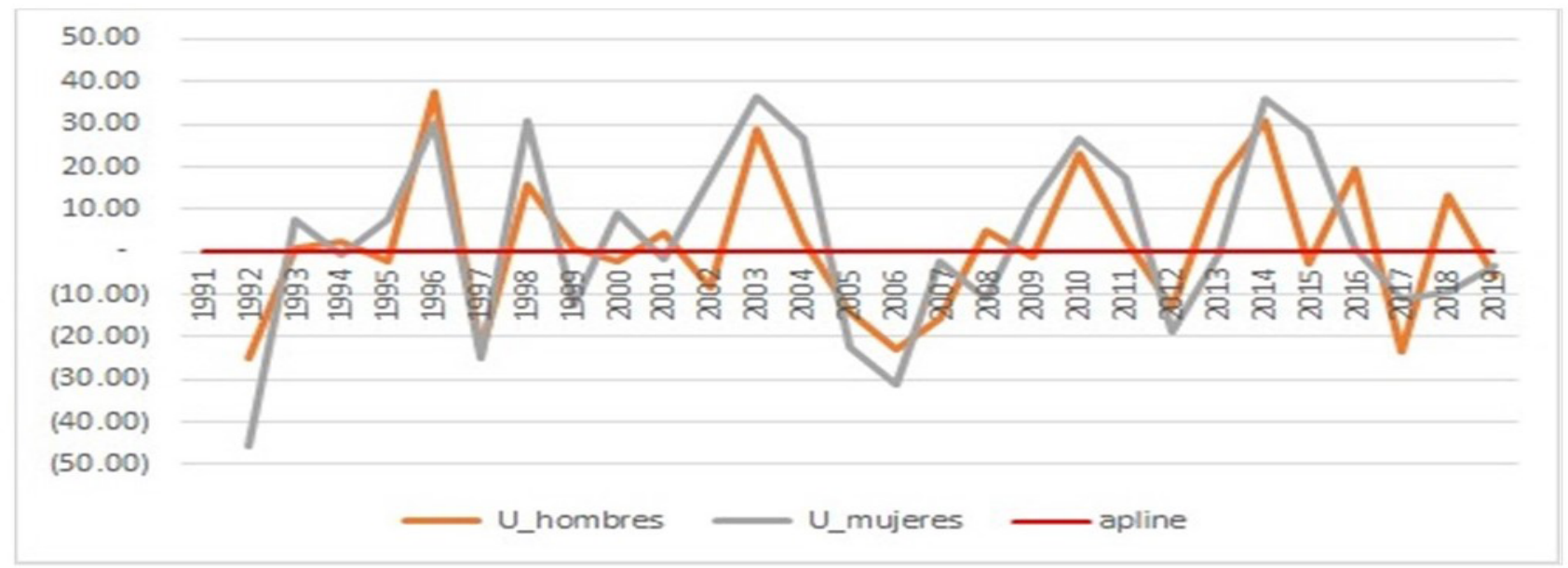

Fuente: Elaboración propia con datos del Banco Mundial (BM).

La Figura2, demuestra que la variación del desempleo masculino es mucho más sensible a las fluctuaciones del mercado laboral en comparación con el sexo femenino. Se logra apreciar que, en los periodos de recesión, las tasas de desempleo entre hombres y mujeres tienden a igualarse, sin embargo, las recuperaciones del sexo masculino son mucho más lentas y se logran en el largo plazo. A continuación, se presentará una tabla en donde se logra apreciar la distribución de los empleos en Honduras por sexo y rama de actividad económica. 
Tabla 1: Distribución del empleo por sexo y rama de actividad

\begin{tabular}{|c|c|c|c|c|c|c|}
\hline \multirow{2}{*}{$\begin{array}{c}\text { Distribución del empleo por sexo y rama de } \\
\text { actividad }\end{array}$} & \multicolumn{2}{|c|}{2016} & \multicolumn{2}{|c|}{2018} & \multicolumn{2}{|c|}{2019} \\
\hline & Hombres & Mujeres & Hombres & Mujeres & Hombres & Mujeres \\
\hline Total Nacional & 100 & 100 & 100 & 100 & 100 & 100 \\
\hline Sector Agricola & 39.8 & 8.4 & 43.8 & 11.1 & 42.8 & 8.3 \\
\hline Agricultura, Silvicultura, Caza y Pesca & 39.5 & 8.4 & 43.6 & 11 & 42.2 & 8.3 \\
\hline Explotacion de minas y canteras & 0.3 & 0 & 0.2 & 0.1 & 0.6 & 0 \\
\hline Sector Industrial & 22.2 & 20 & 21.2 & 18.2 & 22.1 & 19.1 \\
\hline Industria manufacturera & 12.3 & 19.4 & 11.1 & 17.5 & 11.1 & 18 \\
\hline Electricidad, gas y agua & 1.1 & 0.4 & 0.7 & 0.4 & 1.3 & 0.5 \\
\hline Construccion & 8.8 & 0.2 & 9.4 & 0.3 & 9.7 & 0.6 \\
\hline Sector Comercio y Servicios & 37.8 & 71.4 & 35 & 70.7 & 34.8 & 72.6 \\
\hline Comercio por Mayor/Menor, Hoteles/Restaurantes & 17.3 & 37.6 & 14.9 & 36.2 & 15.6 & 37.2 \\
\hline Transp almac y comunic & 5.7 & 1.1 & 6.1 & 1 & 5.4 & 1.1 \\
\hline Estab. finac. seguros, Bienes inmuebles y servicios & 2.4 & 3 & \begin{tabular}{l|l}
2.1 \\
\end{tabular} & 2.4 & 2 & 2.6 \\
\hline Servicios Comunales, Sociales y Personales & 12.4 & 29.7 & 11.9 & 31.1 & 11.8 & 31.7 \\
\hline No especificados & 0.1 & 0.1 & 0| & 0 & 0 & 0 \\
\hline
\end{tabular}

Fuente: Elaboración propia con datos de la Comisión para América Latina y el Caribe (CEPAL).

La tabla 1, permite analizar la brecha ocupacional desde la relación desempleo-PIB (ley de Okun), señalando que, aunque los efectos negativos en el mercado laboral afectan altamente a las mujeres, los sectores que explican en mayor medida el crecimiento económico de Honduras están ocupados por el sexo masculino. Y es que, en el año 2019 la participación masculina en el sector primario alcanzó un (42.8\%), considerando que los empleos adquiridos en este suelen ser muy inestables ante las fluctuaciones del ciclo económico; posicionando a las mujeres con (72.6\%), en el sector terciario o de servicios, identificados como de estabilidad laboral.

\section{Metodología Y FUenTE DE DATOS}

Dada la naturaleza cuantitativa de la investigación, cuyo objetivo primordial pretende cuantificar la incidencia del desempleo por sexo en el crecimiento económico utilizando un alcance de tipo explicativo. Se construirá una base empleando microdatos del Banco Mundial (BM) en el periodo 1991-2019. En consecuencia, se procederá a estimar la ley de Okun utilizando el modelo de primeras diferencias estimado por Mínimos Cuadrados Ordinarios (MCO) tomando como referencia el modelo establecido por Pérez et al, (2014).

A continuación, las ecuaciones a estimar son:

Enfoque Total:

$\Delta U_{t}=\beta_{0}+\beta_{1}\left(\Delta Y_{t}\right)+\varepsilon_{t}$

Enfoque Masculino:

$\Delta U_{H}=\beta_{0}+\beta_{1}\left(\Delta Y_{t}\right)+\varepsilon_{t}$

Enfoque Femenino:

$$
\Delta U_{F}=\beta_{0}+\beta_{1}\left(\Delta Y_{t}\right)+\varepsilon_{t}
$$


donde:

$\beta_{0}=$ Efecto Inmediato en caso de no existir variación del crecimiento económico.

$\beta_{1}=$ Coeficiente de Okun, variación porcentual en desempleo.

$\Delta Y=$ Variación del crecimiento económico real anual.

$\Delta U=$ Variación de la tasa de desempleo abierta.

$\Delta U_{H}=$ Variación de la tasa de desempleo abierta para los hombres.

$\Delta U_{M}=$ variación de la tasa de desempleo abierta para las mujeres.

$e=$ Error de especificación.

Una vez corrido el modelo habrá que prestar atención a los signos de los coeficientes que acompañan a la variable desempleo, ya que si estos presentan signos negativos será un primer indicio de que la ley de Okun aplica a Honduras y en particular a ambos sexos. Los resultados obtenidos sobre el siguiente modelo permitirán comparar los efectos del desempleo por sexo en las variaciones sobre el crecimiento económico real.

\section{Discusión De Resultados}

Una vez analizada la metodología y los datos por utilizar, esta sección muestra los resultados del enfoque empleado para la aplicación de la ley de Okun en la economía hondureña desde una perspectiva del sexo y considerando el efecto total.

En este sentido las Figuras (anexo 1), plantean el análisis inicial de las variables y su comportamiento en el tiempo, proporcionando de forma prematura líneas que permiten analizar la diferencia de cifras entre hombres y mujeres, evidenciando que, durante los períodos de contracción del PIB, el desempleo presenta niveles de disminución mayores para los hombres como fue señalado por Gálvez y Rodríguez (2013), mientras que las mujeres presentan recuperaciones más lentas en el corto plazo.

Para la estimación de los coeficientes se realiza la prueba de raíz unitaria; la tabla 1, presenta los resultados mostrando que todas las series de desempleo son I (0). Esto es así, ya que la hipótesis nula de que la serie no presenta raíz unitaria se acepta en todos los casos y por ende se rechaza la posibilidad que la serie presente primeras diferencias. Lo mismo aplica para la serie del producto, se trata de serie I (O). 
Tabla 2. Prueba de raíz unitaria

\begin{tabular}{lccc}
\hline & Serie & Estadistico & 5\% critico \\
\hline Total Honduras & $\mathrm{U}$ & -29.138 & -12.564 \\
& $\mathrm{Y}$ & -30.567 & -12.564 \\
\hline Hombres & $\mathrm{U}$ & -33.280 & -12.564 \\
& $\mathrm{Y}$ & -30.567 & -12.564 \\
\hline Mujeres & $\mathrm{U}$ & -25.682 & -12.564 \\
& $\mathrm{Y}$ & -30.567 & -12.564 \\
\hline
\end{tabular}

Fuente: Estimaciones propias con base en datos de BM.

A continuación, se presenta la Tabla 2. que muestra el resultado de la prueba de cointegración de Engel y Granger (1987), que muestra que para los tres casos analizados existe una relación de equilibrio de largo plazo entre la tasa de desempleo y el PIB, es decir, las variables están integradas. En este sentido, es posible estimar un modelo econométrico por Mínimos Cuadrados Ordinarios (MCO), teniendo en cuenta la relación de equilibrio de largo plazo.

Tabla 3. Prueba de cointegración

\begin{tabular}{lccc}
\hline & Estadistico & $\mathbf{5 \%}$ critico & Cointegración \\
\hline Total Honduras & -6.508 & -3.572 & $\mathrm{Si}$ \\
\hline Hombres & -7.684 & -3.572 & $\mathrm{Si}$ \\
\hline Mujeres & -5.725 & -3.572 & $\mathrm{Si}$ \\
\hline
\end{tabular}

Fuente: Estimaciones propiascon base en datos de BM.

El paso final consiste en estimar el modelo por MCO para cada uno de los casos analizados. Los resultados indican que, para la estructura de datos utilizada, la ley de Okun por primeras diferencias para la economía de Honduras es estadísticamente no significativa, es decir, que los cambios en el predictor no están asociados con cambios en la respuesta. El resultado reportado es congruente con la evidencia de los autores (Briceño, Dávila y Rojas, 2016), quienes demuestran que en América Latina la relación es negativa, pero es estadísticamente no significativa. 
Tabla 4. Resultados principales

\begin{tabular}{|c|c|c|c|c|c|}
\hline \multicolumn{6}{|c|}{ Ley de Okun } \\
\hline \multicolumn{2}{|l|}{ Enfoque Total } & \multicolumn{2}{|c|}{ Enfoque Masculino } & \multicolumn{2}{|c|}{ Enfoque Femenino } \\
\hline \multicolumn{2}{|c|}{$\begin{array}{c}\mathrm{Ut}=12.602-2.578 \mathrm{Yt} \\
\mathrm{t}(-1.58)(1.72)\end{array}$} & \multicolumn{2}{|c|}{$\begin{aligned} \mathrm{Ut} & =11.419-2.514 \mathrm{Yt} \\
\mathrm{t} & (-1.80)(1.81)\end{aligned}$} & \multicolumn{2}{|c|}{$\begin{aligned} & \mathrm{Ut}=9.8371-1.6740 \mathrm{Yt} \\
& \mathrm{t}(-0.91)(1.19)\end{aligned}$} \\
\hline Pruebas de especificación & Estadistico & Pruebas de especificacion & Estadistico & Pruebas de especificacion & Estadistico \\
\hline R2 & 0.0880 & R2 & 0.1112 & R2 & 0.0309 \\
\hline Portmanteau & 0.7014 & Portmanteau & 0.3222 & Portmanteau & 0.8018 \\
\hline Breusch-Pagan & 0.7722 & Breusch-Pagan & 0.5812 & Breusch-Pagan & 0.3899 \\
\hline LM & 0.3005 & LM & 0.0513 & LM & 0.8883 \\
\hline JB & 0.4948 & JB & 0.4738 & $\mathrm{JB}$ & 0.6224 \\
\hline
\end{tabular}

Fuente: Estimaciones propias con base en datos de BM.

Los resultados obtenidos indican que el coeficiente de Okun, considerando el enfoque total del desempleo en Honduras, tiene el signo adecuado y una magnitud de 2.578 , lo cual indica que por cada aumento del PIB en $1 \%$, la variación de la tasa de desempleo total disminuirá en 2.578 puntos porcentuales.

La aplicación de la ley de Okun concuerda con los datos considerados, aunque no de manera significativa, señalando que el efecto es de 2.514 puntos porcentuales para el caso de los hombres y 1.674 puntos porcentuales para el caso de las mujeres. Evidenciando que la ley de Okun no se cumple para el caso de Honduras, dado que influyen características como el dinamismo, y la volatilidad del mercado laboral.

\section{Conclusiones}

En el presente documento se ha planteado la relación entre la variación de la tasa de desempleo abierta con el crecimiento económico para el caso hondureño y su distinción entre hombres y mujeres mediante el modelo de primeras diferencias de la ley de Okun. Para ello se utilizaron datos de series de tiempo para el periodo comprendido de 1991-2019. En relación a estas consideraciones, el estudio determinó las siguientes conclusiones:

En el análisis estadístico de las variables se destaca la brecha entre hombres y mujeres que presenta el país, en los principales indicadores descritos a lo largo del periodo de tiempo analizado, pues, en ningún momento del periodo descrito se visualiza que la tasa de desempleo abierta para las mujeres sea menor que la de los hombres. De igual forma, se aprecia que la tasa de participación de la mujer en el sector primario y secundario de la economía nunca iguala o supera al sexo masculino. Por lo tanto, se recomienda la implementación y difusión de políticas de igualdad y equidad entre hombres y mujeres, así como la creación de organismos institucionales que velen por el acceso igualitario a los recursos, empleo, ingreso, entre otros.

Del mismo modo, el análisis estadístico de los resultados demuestra que la relación entre la variación de la tasa de desempleo y crecimiento económico es inversa pero estadísticamente no significativa ya que ninguno de los enfoques de la ley de Okun aplica a la economía hondureña. A pesar de ello, se destaca que para el conjunto nacional ante una variación de un punto porcentual en el crecimiento económico supone una desviación en sentido contrario de 2.578 puntos porcentuales; en el caso de los hombres, esta cifra asciende a 2.514, mientras tanto en el caso de las mujeres el valor es de 1.674.

Queda demostrado que el efecto es mayor para el caso de los hombres. Esto sucede ya que el empleo masculino se concentra principalmente en los sectores como el agrícola e industrial que requieren de mucha más mano de obra, sectores que además de explicar en gran medida el crecimiento económico, también son más sensibles a los cambios cíclicos recesivos que provocan en la población masculina variaciones negativas en la cantidad de empleados. Por otro lado, el empleo femenino suele situarse en áreas de comercio y servicios donde el empleo 
es mucho más estable y poseen más derechos laborales.

También se concluye que las características de la economía nacional vinculadas a los empleos conformados principalmente por trabajadores de sectores informales, autoempleo, subempleo y trabajos familiares no remunerados no se reflejan en la TDA nacional; lo anterior sugiere la aplicación de la ley de Okun desde la ampliación de las variables para una valoración en términos de política económica que explique las relaciones del mercado laboral hondureño, desde el análisis de variables que proporcionan resultados significativos.

Para finalizar, cabe mencionar algunas de las limitaciones que presenta el estudio. En primer lugar, el presente trabajo en ningún momento ha planteado la estimación de la ley de Okun mediante el modelo de brechas y ajuste de tendencia y elasticidad, por lo que quedan abiertas nuevas líneas de investigación. En segundo lugar, una de las limitaciones metodológicas que presenta la investigación es la reducida base de datos utilizada debido a la escasez de datos que presenta el país, lo que podría afectar la validez de los resultados.

\section{REFERENCIAS}

Banco Mundial (S.F). Mercado laboral [base de datos]. https://datos.bancomundial.org/pais/honduras

Briceño, M. B., Dávila, G., \& Rojas, M. (2016). Estimación de la Ley de Okun: evidencia empíricapara Ecuador, América Latina y el Mundo. Revista Vista Económica, 1(1), 35-45. https://revistas.unl.edu.ec/index.php/economica/ article/view/209

Castellano López, C. (2013). La Ley de Okun: Por grupos de edad y género. España, Suecia y Reino Unido. https://eprints.ucm.es/id/eprint/25084/1/GECO_2012-13_51126893D_Cristina.pdf

Chamberlin, G. (2011). Okun's law revisited. Economic \& Labour Market Review, 5(2), 104-132.https://linkspringer. com/content/pdf/10.1057/elmr.2011.21.pdf

Comisión Económica para América Latina y el Caribe (S.F). Mercado laboral [base de datos]. https:estadisticas.cepal.org/cepalstat/WEB_CEPALSTAT/buscador.asp

Correa,J.A.J., \&Cervantes,J.M.M.(2017).Laleyde Okún:Una demostración empírica para Colombiay Costa Rica(19922016). Perspectivas, 9(1), 63-72.http://webcache.googleusercontent.com/search?q=cache:RsP9du5ckZAJ:revistas. unicomfacauca.edu.co:106/ojs/index.php/Perspectives/article/download/133/112+\&cd=2\&hl=es419\&ct $=\mathrm{clnk \& gl}=\mathrm{hn}$

Franco Martín, A. C. (2017). Evidencia de la ley de Okun para Colombia, Chile y Argentina: 1980 -2014.https:// repository.ucatolica.edu.co/jspui/bitstream/10983/14428/4/Franco\%202017.\%20Ley\%20de\%20Okun.\%20 Mayo\%209\%20(2).pdf

Gálvez Muñoz, L., \& Rodríguez Modroño, P. (2013). La desigualdad de género en las crisiseconómicas. https://ddd. uab.cat/pub/estudis/2013/181852/desgencris_a2013n07.pdf

Instituto Nacional de Estadística (S.F). Mercado laboral [base de datos]. https://www.ine.gob.hn/V3/baseine/

Knotek II, E. S. (2007). “How useful is Okun's law?”. Economic Review-Federal Reserve Bank of Kansas City. https://search.proquest.com/openview/f95722f4644acdece29467e1d4402f98/1?pq-origsite=gscholar\&cbl=47211

Loría, E., Libreros, C., \& Salas, E. (2012). La ley de Okun en México: una mirada de género, 2000.22011.1. Investigación económica, 71(280), 121-140. http://www.scielo.org.mx/scielo.php?script=sci_ arttext\&pid=S0185-16672012000200005

Méndez, T., Amador, W., Elvir, S., \&Menjívar,J. (2020). BRECHAS DE GÉNERO EN EL MERCADO LABORAL DE HONDURAS 2010-2019. Economía Y Administración (E\&A), 11(1), 9-24. https://doi.org/10.5377/eya. v11i1.10511

Páez, J. 2013. Una revisión de la ley de Okun para Latinoamérica. Universidad del valle; https://bibliotecadigital.univalle.edu.co/bitstream/handle/10893/5961/0461696p.pdf;jsessionid=4FE43B805240D247E73DF7B61CBF71F4? sequence $=1$

Pérez-Aradros Muro, C., Ben Kaabia, M., Montañés Bernal, A. (2014) Evidencia empírica de la ley de Okun en España: 
distinción por sexo y asimetría. https://zaguan.unizar.es/record/17115/files/TAZ-TFG-2014-2297.pd

Porras, S., \& Martín-Román, Á. L. (2020). Relación entre las características de los mercados de trabajo latinoamericanos y la ley de Okun. Serie Documentos de Trabajo; 10/20.

Sánchez Montenegro, N. L., \& Gutiérrez Beltrán, J. F. (2018). Efectos del ciclo económico sobre la brecha de desempleo por género para Colombia durante 2008 a 2017. https:/ciencia.lasalle.edu.co/cgi/viewcontent. cgi?article $=1565 \&$ context $=$ economia

Sánchez-Fung, J. R. (2015). Producto, desempleo y la ley de Okun en la República Dominicana. Ciencia y sociedad, 40(3), 613-637. https://www.redalyc.org/pdf/870/87042736008.pdf

Weller, J., (2012). Crecimiento, empleo y distribución de ingresos en América Latina. Series de la CEPAL, 122, 1-63. https://repositorio.cepal.org/bitstream/handle/11362/5355/S1200557.pdf?sequence=1

\section{Anexo A}
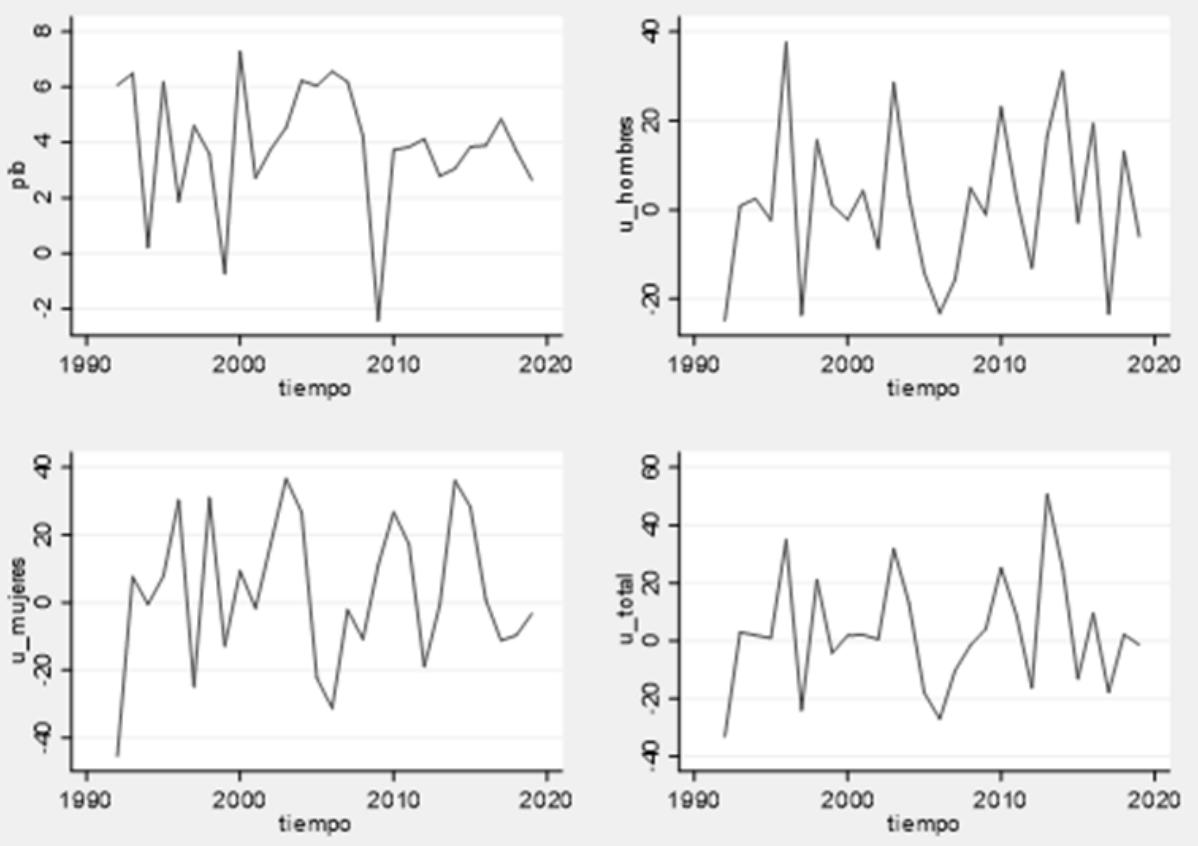

Fuente: Estimaciones propias con base en datos de BM. 